\title{
Quantifying the speed of fluctuations in systolic blood pressure
}

\author{
Arnoldus JR van Gestel ${ }^{1,2}$, Giovanni Camen ${ }^{1}$, Christian F Clarenbach ${ }^{1}$, Noriane Sievi ${ }^{1}$, \\ Valentina A Rossi ${ }^{1}$ and Malcolm Kohler ${ }^{1,3}$
}

Increased blood pressure variability (BPV), even in the absence of hypertension, has been identified as an important independent cardiovascular risk factor (CVRF). However, the role of the speed of changes in systolic blood pressure (SBP; vSBP) on cardiovascular risk needs to be investigated. The objective of this study was to investigate whether subjects with a high cardiovascular risk profile have an increased degree and speed of changes in SBP compared with subjects with low or no risk. Resting beat-to-beat blood pressure (BP) was recorded for $5 \mathrm{~min}$. Standard BPV measures in both time and frequency domains were conducted. The s.d. of SBP (s.d.-SBP) values was used to quantify the degree of BPV. vSBP was assessed by calculating the slopes of oscillatory fluctuations in SBP for different interbeat intervals (IBI). Subjects were allocated to one of four groups according to the number of CVRFs ( $0,1,2, \geqslant 3$ CVRF). Of 122 subjects, $19.7 \%$ had 0 CVRF, $27.0 \%$ had $1,32.0 \%$ had 2 and $21.3 \%$ had $\geqslant 3$ CVRFs. There was an increase in vSBP across the four risk groups. The vSBP in patients without CVRF was 3.12 (1.09), 1 CVRF 3.23 (1.07), 2 CVRF 4.16 (2.26) and $\geqslant 3$ CVRF $4.22(1.66 ; P=0.015)$. The s.d.-SBP was not significantly different between the cardiovascular risk groups. The speed of fluctuations in SBP rather than the degree of BPV is pronounced in patients with elevated cardiovascular risk. Increased speed of BP fluctuations may thus be a contributing mechanism to cardiovascular morbidity.

Hypertension Research (2013) 36, 1039-1044; doi:10.1038/hr.2013.62; published online 20 June 2013

Keywords: blood pressure variability; cardiovascular risk; systolic blood pressure

\section{INTRODUCTION}

The high prevalence of cardiovascular disease may be attributable to risk factors such as obesity, diabetes, dyslipidemia and hypertension, and may be linked to common lifestyle determinants such as diet, physical activity and tobacco consumption. ${ }^{1}$ It is widely known that increased systolic blood pressure (SBP) is a powerful risk factor for cardiovascular events. However, there is still no consensus as to how hypertension leads to cardiovascular events. ${ }^{2}$

Blood pressure variability (BPV) is a measure of cardiovascular and autonomic dysfunction that is used for research purposes. ${ }^{3-6}$ Increased visit-to-visit BPV, even in the absence of sustained hypertension, has been identified as an important independent cardiovascular risk factor (CVRF) and is associated with both the prevalence and severity of target-organ damage. ${ }^{7-9}$ This has been demonstrated in both the general population and hypertensive patients. ${ }^{7-9}$ In addition, Rothwell et al. ${ }^{9,10}$ found that even increased BPV over a few minutes has some prognostic value.

Several invasive and noninvasive techniques have been proposed to quantify the fluctuations in SBP. BPV is usually expressed as the s.d. of average ambulatory blood pressure (BP) values over $24 \mathrm{~h}$, as well as during daytime and nighttime periods. ${ }^{4,11,12}$ However, the usefulness of the s.d. is limited as it only reflects the dispersion of SBP measurements around the mean not accounting for the time rate at which the changes in BP take place. As biological tissue is especially susceptible to damage by sudden changes of pressure, the speed of changes in SBP per se may be a major determinant of target-organ damage. ${ }^{13-18}$

A more reliable representation of BPV may be achieved by calculating the mean of the differences between intermittent BP measurements (average real variability, ARV) using data from $24 \mathrm{~h}$ SBP monitoring. ${ }^{19}$ ARV reflects SBP dynamics over time and overcomes the pitfalls of the commonly used s.d. However, ARV does not reflect short-term changes in SBP, which may be an important measure regarding target-organ damage. ${ }^{20}$

Data from studies investigating the relationship between the speed of SBP fluctuations and cardiovascular risk are currently not available. Therefore, the aim of the present study was to investigate whether subjects with elevated cardiovascular risk experience faster changes in SBP than subjects with low or no cardiovascular risk, using a new signal-analysis method.

\section{METHODS}

Subjects

A group of 122 subjects with a heterogenous cardiovascular risk profile including 59 patients with chronic obstructive pulmonary disease, 30 patients

${ }^{1}$ Pulmonary Division, University Hospital of Zurich, Zurich, Switzerland; ${ }^{2}$ Department of Health, Zurich University of Applied Sciences, Winterthur, Switzerland and ${ }^{3}$ Center for Integrative Human Physiology, University of Zurich, Zurich, Switzerland

Correspondence: Professor Dr AJR van Gestel, Department of Health, Zurich University of Applied Sciences, Technikumstrasse 71, CH-8401 Winterthur, Switzerland. E-mail: vrns@zhaw.ch

Received 7 January 2013; revised 21 March 2013; accepted 14 April 2013; published online 20 June 2013 
with obstructive sleep apnea and 33 healthy subjects of both sexes aged between 18 and 75 years were included. In order to investigate a group of subjects with a heterogenous cardiovascular risk profile, we did not exclude patients with a history of coronary artery disease or stroke. However, no patients with acute myocardial infarction or stroke were included. The patients were referred to the Pulmonary Division, University Hospital of Zurich, Switzerland between February 2010 and April 2011. Healthy volunteers were recruited from among the hospital staff and college students. The study was approved by the Research Ethics Committee of the University Hospital of Zurich, Switzerland (EK-1734, EK-1600) and written informed consent was obtained from all subjects.

\section{Blood pressure}

BP was measured in triplicate with a semiautomated oscillometric device (Omron Healthcare, Kyoto, Japan) separated by 1-min intervals after resting in supine position for $10 \mathrm{~min}$ in a temperature-controlled, quiet, dim room, with the study subject in supine position. All BP measurements were taken on the upper right arm.

\section{BP variability}

Noninvasively obtained continuous hemodynamic measurements of beatto-beat arterial BP were recorded with the Finometer device (Finapress Measurement Systems, Amsterdam, The Netherlands) using a photoplethysmographic-arterial/volume clamp applied at the level of the right index finger. ${ }^{11,21}$ The patients were instructed to breath regularly.

The Finometer device has been validated against intra-arterial measurements and has been shown to accurately demonstrate BP trends ${ }^{12}$ and estimate $24 \mathrm{~h}$ BPV. ${ }^{22}$ Furthermore, the Finometer has been validated using the Rica-Rocci Korotkoff method..$^{23}$ The Finometer corrects for the hydrostatic height of the finger with respect to the heart level. ${ }^{24}$ The peak SBP was used to calculate the interbeat intervals (IBI) in milliseconds. For statistical analysis, the beat-to-beat values of SBP were evaluated from 5-min beat-to-beat recordings, that is, from 300 to 400 SBP values $(n)$

The following standard time domain measures of BPV were computed: mean SBP in $\mathrm{mm} \mathrm{Hg}$ and the root mean square of successive difference of beat-to-beat SBP values (rMSSD-SBP) in $\mathrm{mm} \mathrm{Hg}$. The s.d. of SBP values intervals (s.d.-SBP) in $\mathrm{mm} \mathrm{Hg}$ was used to quantify the degree BPV.

Standard frequency-domain measures of BPV were computed using fast Fourier transformation with BioTrace + software $^{25}$ The power spectrum of beat-to-beat BPV consists of the following bands: low-frequency spectral power $(0.04-0.15 \mathrm{~Hz})$ and high-frequency spectral power $(0.15-0.45 \mathrm{~Hz}) .^{26,27}$ Power was calculated in percentage of total power (\%) and in absolute values of power $\left(\mathrm{mm} \mathrm{Hg}^{2}\right)$.

A new signal-analysis technique was developed to quantify the speed of changes in SBP (vSBP) using beat-to-beat SBP values. The vSBP was determined by the following procedure:

The delta of changes in SBP over one IBI $\left(\Delta \mathrm{SBP}_{1}\right)$ was calculated using the following formula:

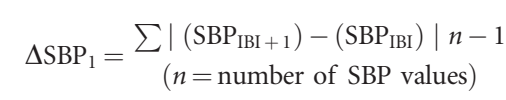

the delta of changes in SBP over two IBIs $\left(\mathrm{vSBP}_{2}\right)$ was calculated using the following formula:

$$
\Delta \mathrm{SBP}_{2}=\begin{aligned}
& \sum\left|\left(\mathrm{SBP}_{\mathrm{IBI}+2}\right)-\left(\mathrm{SBP}_{\mathrm{IBI}}\right)\right| n-1 \\
& (n=\text { number of SBP values })
\end{aligned}
$$

the average $\mathrm{vSBP}_{2}$ was quantified by dividing the $\triangle \mathrm{SBP}_{2}$ by the number of IBIs (two).

$$
\mathrm{vSBP}_{2}=\Delta \mathrm{SBP}_{2} / 2
$$

we followed the same procedure for determining the average speed of changes in SBP over $x$ IBIs (from 1-24 IBI).

To test the intra-subject reproducibility of the IBI measurement, a second $5 \mathrm{~min}$ Finapres recording, which was done $10 \mathrm{~min}$ after the first one, was analyzed.

\section{Cardiovascular risk assessment}

Risk factors were defined as a body mass index $>25 \mathrm{~kg} \mathrm{~m}^{-2}$ (World Health Organization definition of overweight), arterial hypertension with a BP $>140 / 90 \mathrm{~mm} \mathrm{Hg},{ }^{28}$ a cholesterol level $>5 \mathrm{mmoll}^{-1}{ }^{29}$ a fasting blood glucose level $>7 \mathrm{mmoll}^{-130}$ and active smoking. To investigate whether subjects with elevated cardiovascular risk have an increased speed of changes in SBP compared with subjects with low or no risk, subjects were grouped according to the number of risk factors. ${ }^{31}$

\section{Data analysis}

A statistical software package was used for all calculations (SPSS for Windows, version 11.0, SPSS, Chicago, IL, USA). Descriptive data for continuous variables are expressed as mean and s.d.. We used repeated measures to test the equality of means of beat-to-beat SBP changes with correlation analysis. For further analyses, the subjects were grouped according to the number of CVRFs and allocated to four groups (0 CVRF, 1 CVRF, 2 CVRF and $\geqslant 3$ CVRF). Differences between groups were evaluated by using analysis of variance. A $P$-value of $<0.05$ was considered to indicate statistical significance.

\section{RESULTS}

\section{Patients' characteristics}

Table 1 shows the anthropometrical characteristics and the cardiovascular risk profile of the 122 subjects (32 females). Significant differences between groups were observed for the variables age, weight, body mass index, smoking pack years, mean diastolic and mean arterial BP (Table 2).

According to the Joint National Committee on Prevention, Detection, Evaluation, and Treatment of High Blood Pressure guidelines, ${ }^{32}$ 59 patients $(48.4 \%)$ had a normal SBP, 49 patients $(40.2 \%)$ had prehypertension, 11patients $(9.0 \%)$ had hypertension stage 1 and 3 patients $(2.4 \%)$ had hypertension stage 2 .

\section{The vSBP}

An example of beat-to-beat variability of SBP in a patient with three CVRFs is shown in Figure 1. The values reflecting the speed of changes in SBP for different IBIs are shown in Table 3. There were significant differences between the groups regarding the $\mathrm{vSBP}_{1}$ and $\mathrm{vSBP}_{2}$ Table 3). The $\mathrm{vSBP}_{2}$ in the four cardiovascular risk groups is shown in Figure 2.

\section{Table 1 Characteristics of the subjects}

\begin{tabular}{lc}
\hline & Study subjects \\
\hline Number of subjects & 122 \\
Male/female & $90 / 32$ \\
Age (years) & $53.3(16.4)$ \\
Height (cm) & $172.9(8.6)$ \\
Weight (kg) & $81.6(20.3)$ \\
BMI (kg m ${ }^{-2}$ ) & $27.2(6.0)$ \\
Pack years ( $n$ ) & $27.5(28.7)$ \\
Hypertension (\%) & 10.6 \\
Active smoking (\%) & 16.3 \\
Overweight (\%) & 57.7 \\
Diabetes (\%) & 11.0 \\
Hypercholesterolemia (\%) & 40.7 \\
Systolic BP (mm Hg) & $124.2(36.8)$ \\
Diastolic BP (mm Hg) & $74.6(11.4)$ \\
Arterial BP (mm Hg) & $91.4(11.8)$ \\
Heart rate (min ${ }^{-1}$ ) & $71.7(13.9)$ \\
\hline
\end{tabular}

Abbreviations: BMI, body mass index; $\mathrm{BP}$, blood pressure Data are presented as mean (s.d.). Hypertension, BP $>140 / 90 \mathrm{~mm} \mathrm{Hg}$; overweight, $\mathrm{BMI}>25 \mathrm{~kg} \mathrm{~m}^{-2}$; diabetes, fasting blood glucose level $>7 \mathrm{mmol} \mathrm{I}^{-1}$; hypercholesterolemia, cholesterol level $>5 \mathrm{mmoll}^{-1}$. 


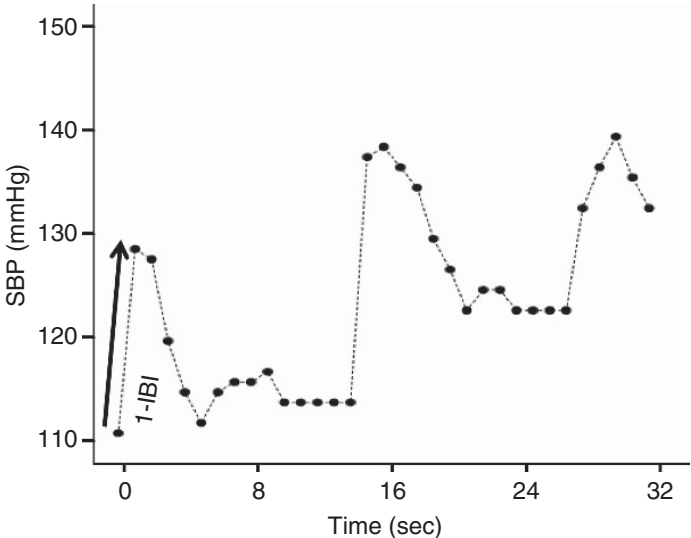

Figure 1 An example of beat-to-beat variability of systolic blood pressure (SBP) over $32 \mathrm{~s}$ in a patient with three cardiovascular risk factors (CVRFs). The arrow demonstrates a $\triangle \mathrm{SBP}$ value of $18 \mathrm{~mm} \mathrm{Hg}$ after one IBI.

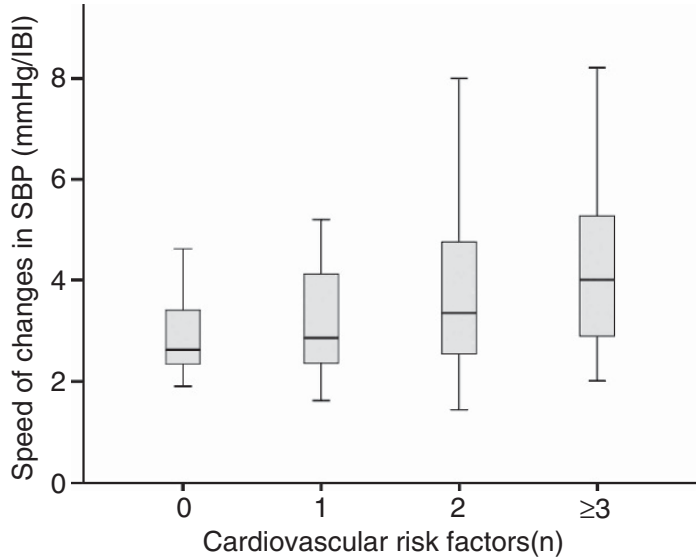

Figure 2 Box plots showing median, the 25th and 75th percentile of the speed of changes in SBP (vSBP) for one IBI of the subjects grouped according to the number of cardiovascular risk factors (CVRFs; 0 CVRF: median=2.67, 1 CVRF: median=2.87, 2 CVRF: median=3.43, $\geqslant 3$ VCRF: median $=4.01, P=0.015$ ).

Table 2 Characteristics of the CVR-groups

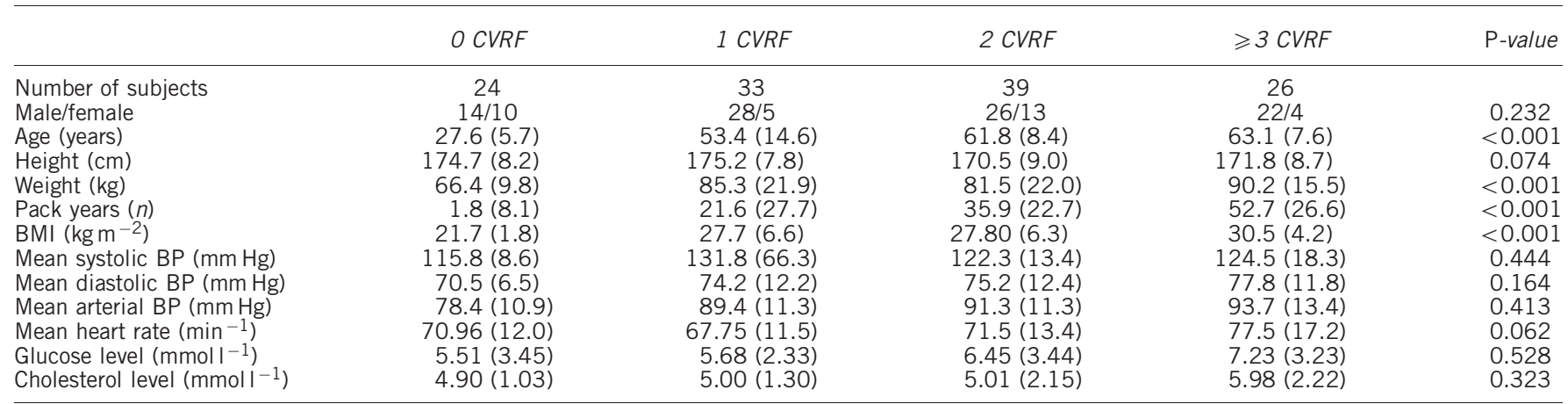

Abbreviations: BMI, body mass index; BP, blood pressure; CVRF, cardiovascular risk factor.

Data are presented as mean (s.d.). P-value comparing the four groups (analysis of variance, ANOVA).

Groups stratified by the number of CVRFs.

Table 3 The speed of changes in SBP

\begin{tabular}{|c|c|c|c|c|c|}
\hline & O CVRF & $1 \mathrm{CVRF}$ & $2 \mathrm{CVRF}$ & $\geqslant 3 \mathrm{CVRF}$ & P-value \\
\hline $\mathrm{vSBP}_{1}$ & $3.12(1.09)$ & $3.23(1.07)$ & $4.16(2.26)$ & $4.22(1.66)$ & 0.015 \\
\hline $\mathrm{VSBP}_{2}$ & $2.17(0.87)$ & $2.23(0.76)$ & $2.78(1.41)$ & $2.94(1.18)$ & 0.020 \\
\hline $\mathrm{vSBP}_{3}$ & $1.68(0.83)$ & $1.65(0.54)$ & $1.91(0.94)$ & $2.02(0.94)$ & 0.269 \\
\hline $\mathrm{vSBP}_{4}$ & $1.42(0.74)$ & $1.33(0.41)$ & $1.49(0.74)$ & $1.65(0.82)$ & 0.333 \\
\hline $\mathrm{vSBP}_{5}$ & $1.24(0.66)$ & $1.14(0.37)$ & $1.25(0.61)$ & $1.46(0.65)$ & 0.205 \\
\hline $\mathrm{vSBP}_{6}$ & $1.08(0.54)$ & $1.02(0.35)$ & $1.08(0.45)$ & $1.19(0.44)$ & 0.556 \\
\hline $\mathrm{vSBP}_{9}$ & $0.74(0.26)$ & $0.74(0.27)$ & $0.76(0.31)$ & $0.83(0.25)$ & 0.554 \\
\hline $\mathrm{vSBP}_{12}$ & $0.74(0.23)$ & $0.74(0.21)$ & $0.76(0.22)$ & $0.65(0.25)$ & 0.675 \\
\hline $\operatorname{vSBP}_{15}$ & $0.49(0.19)$ & $0.48(0.17)$ & $0.49(0.18)$ & $0.55(0.17)$ & 0.548 \\
\hline $\mathrm{vSBP}_{18}$ & $0.42(0.16)$ & $0.41(0.14)$ & $0.42(0.16)$ & $0.47(0.16)$ & 0.481 \\
\hline $\mathrm{vSBP}_{21}$ & $0.37(0.15)$ & $0.35(0.12)$ & $0.36(0.13)$ & $0.40(0.15)$ & 0.608 \\
\hline $\mathrm{vSBP}_{24}$ & $0.33(0.13)$ & $0.32(0.11)$ & $0.31(0.11)$ & $0.35(0.13)$ & 0.498 \\
\hline
\end{tabular}

Abbreviations: CVRF, cardiovascular risk factor; IBI, interbeat intervals; SBP, systolic blood pressure; $\mathrm{vSBP}_{\mathrm{x}}(\mathrm{mm} \mathrm{Hg} / \mathrm{IBI})$, the speed of changes in SBP for $\mathrm{x}$ IBIs (Equation 2). Data are presented as mean (s.d.)

$P$-value comparing the four groups (analysis of variance, ANOVA)

Groups stratified by the number of CVRFs.
Intra-subject reproducibility of the average vSBP

There were excellent agreements between the two measurements of average vSBP for the different IBIs: $\mathrm{vSBP}_{1} r=0.93(P<0.001)$ and $\mathrm{vSBP}_{2} r=0.83(P<0.001)$.

\section{Time and frequency-domain measures of BPV}

In Table 4, the more widely established sophisticated measures reflecting BPV in time and frequency-domain and their correlations with $\mathrm{vSBP}_{2}$ are presented. There was a significant correlation between $\mathrm{vSBP}_{2}$ and mean SBP, s.d.-SBP, rMSSD-SBP, low-frequency spectral power and high-frequency spectral power.

\section{The degree of changes in SBP}

In Table 5, measures reflecting BPV in time and frequency-domain of the different cardiovascular risk groups are presented. There were no significant differences between the groups regarding the degree of changes in SBP. 


\section{DISCUSSION}

To the best of our knowledge, this is the first study assessing both the degree and the speed of fluctuations in SBP in a group of subjects with a heterogenous cardiovascular risk profile using beat-to-beat recordings of SBP. We found that the speed of fluctuations in SBP rather than the degree of BPV is pronounced in patients with elevated cardiovascular risk.

There is growing evidence that BPV, even in the absence of sustained hypertension, is an independent predictor of organ damage and cardiovascular events. ${ }^{7-9,33-37}$ BPV is usually estimated by the s.d. or coefficient of variation of average ambulatory SBP values over $24 \mathrm{~h}$. As these conventional estimates of BPV have limited predictive power compared with other more traditional CVRFs, Bilo and Parati ${ }^{20}$ underlined the clinical importance of refining the quantification of BPV. As BPV includes both short-term and circadian components, the s.d. of average ambulatory SBP values over $24 \mathrm{~h}$ is a very rough indicator of the true BP dynamics. In addition, the s.d. of average ambulatory SBP values over $24 \mathrm{~h}$ is influenced by the pronounced nocturnal fall in BP and the responses to environmental stressors. Furthermore, the s.d.-SBP values mainly reflects the degree of SBP fluctuations not accounting for the time rate at which the changes in SBP over the $24 \mathrm{~h}$ take place. To address these limitations, several new methods have been developed in order to refine the estimation of BPV and get quantifiable information on the speed of changes in SBP..$^{19,33,38}$

In 2005, a new method to quantify the speed of changes in SBP (ARV) was proposed by Mena et al. ${ }^{19}$ ARV was quantified by calculating the mean of the differences between intermittent BP measurements using data from $24 \mathrm{~h}$ ambulatory BP monitoring. ${ }^{19}$ Accordingly, in a large study including 8938 subjects from 11 populations, ARV was found to be a better predictor of mortality and cardio- and cerebrovascular events than the s.d. ${ }^{33}$ In 2005,

\section{Table 4 Correlations with vSBP 2}

\begin{tabular}{lrr}
\hline Variable & Coefficient r & P-value \\
\hline SBP (mm Hg) & 0.28 & 0.031 \\
s.d.-SBP (mm Hg) & 0.60 & $<0.001$ \\
rMSSD-SBP (mm Hg) & 0.83 & $<0.001$ \\
LF-power (\%) & 0.009 & 0.932 \\
HF-power (\%) & -0.012 & 0.910 \\
LF-power (mm Hg & 0.025 \\
HF-power $\left(\mathrm{mm} \mathrm{Hg}^{2}\right)$ & 0.243 & 0.025 \\
\end{tabular}

Abbreviations: HF-power, high-frequency spectral power; IBI, interbeat intervals; LF-power, low-frequency spectral power; rMSSD-SBP, the root mean square successive differences of SBP; SBP, systolic blood pressure; SBP, mean of the SBP values; s.d.-SBP, s.d. of SBP values; $\mathrm{VSBP}_{2}$, the speed of changes in SBP after two IBIs (Equation 2).

Correlation is expressed as Pearson's correlation coefficient $(r)$. VLF $=0.02-0.05 \mathrm{~Hz}$; $\mathrm{LF}=0.05-0.15 \mathrm{~Hz} ; \mathrm{HF}=0.15-0.4 \mathrm{~Hz}$.
Zakopoulos et al. ${ }^{39}$ proposed the 'time rate of BPV' in order to measure how fast or how slow and in which direction SBP values change. The 'time rate of BPV' was derived from $24 \mathrm{~h}$ ambulatory BP monitoring and was defined as the first derivative of the SBP values against time. ${ }^{39}$ Manios et al. ${ }^{40}$ used this index to quantify short-term changes in SBP in 162 normotensive patients with suspected coronary artery disease. The authors found that the 'time rate of BPV' is more pronounced in patients with coronary atherosclerotic lesions compared with controls with normal angiography. ${ }^{40}$ However, the use of ambulatory BP monitoring has been challenged because the time between two consecutive measurements is too long to obtain information on the SBP changes occurring between the subsequent measurements. ${ }^{20}$ To gain information about short-term changes in SBP, it may be more accurate to use a sampling interval that is no longer than the beat-to-beat interval.

Attempts to quantify the speed of SBP changes over short-time intervals are justified by the results of Mancia et al..$^{38}$ The authors demonstrated that the slope of the rapid-and short-duration changes in SBP was significantly and markedly greater in hypertensive subjects than in normotensive individuals. ${ }^{38}$ To address the above-mentioned issues, in this study we used a standardized clinical setting and analyzed $5 \mathrm{~min}$ of beat-to-beat recordings of SBP. By calculating the slopes of oscillatory fluctuations in SBP for different IBIs, we quantified the speed of SBP (vSBP) changes. We found that the vSBP in subjects considered to be at a high cardiovascular risk was significantly higher than in patients with no or low cardiovascular risk. This finding suggests that with advanced cardiovascular risk, SBP is more prone to sudden and marked changes. Interestingly, we found that the degree of BPV as quantified by s.d.-BPV was not associated with cardiovascular risk. These findings may underline the importance of the speed of changes in SBP rather than the amount of BPV in cardiovascular disease.

BPV has been found to be increased in conditions characterized by dysfunction of the cardiovascular autonomic nervous system and reduced responsiveness of the baroreceptor reflex to transient rise of BP. ${ }^{6}$ The clinical importance of sudden fluctuations in SBP has been underlined by the fact that there is an increased incidence of acute cardiovascular events (for example, stroke, acute myocardial infarction) in the morning hours at the time of the pressor effect of arousal, mental stress and anger, irregular heavy physical exertion and sexual activity. ${ }^{41-43}$ These findings may have clinical implications because the speed of SBP changes, rather than the amount of BPV, may represent the damaging component of BPV. ${ }^{14-16,44}$ Fast changes in SBP may cause acute oscillatory shear stress on the vascular wall and may therefore facilitate endothelial dysfunction and initiation of atherosclerosis. ${ }^{17,40}$ As vascular endothelial damage is induced by radial and axial shear stresses, it may be reasonable to postulate that

Table 5 Time and frequency-domain measures of BPV

\begin{tabular}{|c|c|c|c|c|c|}
\hline$|B|(\mathrm{n})$ & O CVRF & 1 CVRF & $2 C V R F$ & $\geqslant 3 \mathrm{CVRF}$ & P-value \\
\hline s.d.-SBP (mm Hg) & $7.45(3.30)$ & $7.20(2.99)$ & $7.19(2.31)$ & $7.66(2.27)$ & 0.938 \\
\hline rMSSD-SBP (mm Hg) & $2.18(1.34)$ & $1.85(1.00)$ & $1.87(0.95)$ & $2.14(1.01)$ & 0.628 \\
\hline LF-power (\%) & 38.21 (11.98) & $38.03(13.67)$ & $38.74(13.91)$ & $40.53(16.12)$ & 0.939 \\
\hline HF-power (\%) & 51.39 (17.96) & $52.17(22.48)$ & $51.74(19.09)$ & $51.31(18.97)$ & 0.999 \\
\hline LF-power (mm Hg${ }^{2}$ ) & $8.05(8.54)$ & $8.67(11.84)$ & $5.57(5.07)$ & $6.74(6.77)$ & 0.621 \\
\hline HF-power $\left(\mathrm{mm} \mathrm{Hg}^{2}\right)$ & $3.52(3.09)$ & $4.24(4.96)$ & $3.57(2.23)$ & $4.26(2.08)$ & 0.811 \\
\hline
\end{tabular}

Abbreviations: BPV, blood pressure variability; CVRF, cardiovascular risk factor; HF-power, high-frequency spectral power; IBI, interbeat intervals; LF-power, low-frequency spectral power; rMSSDAbbreviations: BPV, blood pressure variability; CVRF, cardiovascular risk factor; HF-power, high-frequency spectral pow
SBP, the root mean square successive differences of SBP; SBP, systolic blood pressure; s.d.-SBP, s.d. of SBP values.

Data are presented as mean (s.d.). VLF $=0.02-0.05 \mathrm{~Hz} ; \mathrm{LF}=0.05-0.15 \mathrm{~Hz} ; \mathrm{HF}=0.15-0.4 \mathrm{~Hz}$. P-value comparing the four groups (analysis of variance, ANOVA)

Groups stratified by the number of CVRFs. 
BP deviations in either direction are harmful. ${ }^{13}$ Chappell et al. ${ }^{17}$ found that fast SBP changes induce expression of endothelial cell leukocyte adhesion molecules, and, presumptively, migration into the arterial wall. In addition, sudden oscillatory fluctuations of SBP may cause variation of intravascular pressure on the vessel wall, resulting in vascular remodeling and hardening of blood vessels by atherosclerosis. ${ }^{17-19,40}$ Fast fluctuations of SBP may also have a paramount effect on cerebrovascular function. Sudden decreases in SBP may cause cerebral ischemia, ${ }^{45,46}$ sudden increases can cause cerebral hemorrhage, ischemic stroke $^{46,47}$ and spasm in larger cerebral arteries. ${ }^{46,48}$

The vSBP is a new index for reflecting the speed of changes in SBP that can easily be obtained in routine clinical practice and may be regarded as a useful additional step toward a better understanding of the characteristics of BPV. In addition, we found that the intra-subject reproducibility of the vSBP showed good agreement and significant correlations with the conventional measurements of BPV in both the time and frequency domains. We therefore recommend using the vSBP by calculating the slopes of oscillatory fluctuations in SBP for different IBIs in future studies on this topic.

The present study has certain limitations that need to be taken into account. The design of this study does not allow to establish a causal relationship between the speed of SBP fluctuations and cardiovascular disease. Increased BPV often clusters with established CVRFs, such as older age, hypertension and diabetes mellitus. Increased BPV is associated with the prevalence of acute cardiovascular events, but cardiovascular disease at the same time increases BPV. This makes disentangling the independent effects of CVRFs on BPV a daunting task. Finally, more studies on beat-to-beat SBP measurements in different cohorts of patients at cardiovascular risk are needed to assess its clinical usefulness.

In conclusion, we found that the speed of fluctuations in SBP, rather than the degree of $\mathrm{BPV}$, is pronounced in patients with elevated cardiovascular risk. Increased speed of BP fluctuations may thus be a contributing mechanism to cardiovascular morbidity.

Author contributions: Professor Dr rer. medic. Arnoldus JR van Gestel has made substantial contribution to conception and design, acquisition of data, analysis and interpretation of data, has drafted the submitted article or revised it critically for important intellectual content, and has provided final approval of the version to be published and is guarantor of the study; Dr med. Giovanni Camen, MD, Noriane A Sievi and Dr med. Valentina A Rossi have made substantial contribution to conception and design, acquisition of data, analysis and interpretation of data, and have drafted the submitted article and revised it critically for important intellectual content; Dr med. Christian F Clarenbach, MD and Professor Dr med. Malcolm Kohler, MD have made substantial contribution to conception and design, acquisition of data, analysis and interpretation of data, have drafted the submitted article and revised it critically for important intellectual content, and have provided final approval of the version to be published.

1 Yusuf S, Reddy S, Ounpuu S, Anand S. Global burden of cardiovascular diseases: part I: general considerations, the epidemiologic transition, risk factors, and impact of urbanization. Circulation 2001; 104: 2746-2753.

2 Rothwell P. Limitations of the usual blood-pressure hypothesis and importance of variability, instability, and episodic hypertension. Lancet 2010; 375: 938-948.

3 Mancia G, Parati G, Pomidossi G, Casadei R, Di Rienzo M, Zanchetti A. Arterial baroreflexes and blood pressure and heart rate variabilities in humans. Hypertension 1986; 8: 147-153.

4 Mancia G, Ferrari A, Gregorini L, Parati G, Pomidossi G, Bertinieri G, Grassi G, di Rienzo M, Pedotti A, Zanchetti A. Blood pressure and heart rate variabilities in normotensive and hypertensive human beings. Circ Res 1983; 53: 96-104.

5 Singh RB, Cornélissen G, Weydahl A, Schwartzkopff O, Katinas G, Otsuka K, Watanabe Y, Yano S, Mori H, Ichimaru Y, Mitsutake G, Pella D, Fanghong L, Zhao Z, Rao RS, Gvozdjakova A, Halberg F. Circadian heart rate and blood pressure variability considered for research and patient care. Int J Cardiol 2003; 87: 9-28.
6 Se-Joong Rim. Blood pressure variation and cardiovascular risks. Korean Circ J 2008; 38: 131-134.

7 Parati G, Faini A, Valentini M. Blood pressure variability: its measurement and significance in hypertension. Curr Hypertens Rep 2006; 8: 199-204.

8 Grove JS, Reed DM, Yano K, Hwang LJ. Variability in systolic blood pressure: a risk factor for coronary heart disease? Am J Epidemiol 1997; 145: 771-776.

9 Rothwell PM, Howard SC, Dolan E, O'Brien E, Dobson JE, Dahlöf B, Sever PS Poulter NR. Prognostic significance of visit-to-visit variability, maximum systolic blood pressure, and episodic hypertension. Lancet 2010; 375: 895-905.

10 Rothwell PM, Howard SC, Dolan E, ASCOT-BPLA and MRC Trial Investigators. Effects of beta blockers and calcium-channel blockers on within-individual variability in blood pressure and risk of stroke. Lancet Neurol 2010; 9: 469-480.

11 Kurki T, Smith NT, Head N, Dec-Silver H, Quinn A. Noninvasive continuous blood pressure measurement from the finger: optimal measurement conditions and factors affecting reliability. J Clin Monit 1987; 3: 6-13.

12 Imholz BP, Wieling W, Montfrans GA, Wesseling KA. Fifteen years experience with finger arterial pressure monitoring. Cerebrovasc Res 1998; 38: 605-616.

13 MacKenzie ET, Strandgaard S, Graham DI, Jones JV, Harper AM, Farrar JK. Effects of acutely induced hypertension in cats on pial arteriolar caliber, local cerebral blood flow, and the blood-brain barrier. Circ Res 1976; 39: 33-41.

14 Mancia G, Parati G, Di Rienzo M, Zanchetti A. Blood pressure variability. In: Zanchetti $A$ and Mancia G (eds). Handbook of hypertension Vol 17 Pathophysiology of hypertension. AmsterdamElsevier Science, 1997, pp 117-169.

15 Tatasciore A, Renda G, Zimarino M, Soccio M, Bilo G, Parati G, Schillaci G, De Caterina R. Awake systolic blood pressure variability correlates with target-organ damage in hypertensive subjects. Hypertension 2007; 50: 325-332.

16 Palatini P, Penzo M, Racioppa A, Zugno E, Guzzardi G, Anaclerio M, Pessina AC. Clinical relevance of nighttime blood pressure and of daytime blood pressure variability. Arch Intern Med 1992; 152: 1855-1860.

17 Chappell DC, Varner SE, Nerem RM, Medford RM, Alexander RW. Oscillatory shear stress stimulates adhesion molecule expression in cultured human endothelium. Circ Res 1998; 82: 532-539.

18 De Keulenaer GW, Chapell DC, Ishikaza N, Nerem RM, Alexander RW, Griendling KK. Oscillatory and steady laminar shear stress differentially affect human endothelial redox state: role of a superoxide-producing NADH oxidase. Circ Res 1998; 82: 1094-1101.

19 Mena L, Pintos S, Queipo NV, Aizpúrua JA, Maestre G, Sulbarán T. A reliable index for the prognostic significance of blood pressure variability. J Hypertens 2005; 23: 505-511.

20 Bilo G, Parati G. Rate of blood pressure changes assessed by $24 \mathrm{~h}$ ambulatory blood pressure monitoring: another meaningful index of blood pressure variability? J Hypertens 2011; 29: 1054-1058.

21 Smith NT, Wesseling KW, De Wit B. Evaluation of two prototype devices producing noninvasive pulsatile calibrated blood pressure from a finger. J Clin Monit 1985; 1: 17-29.

22 Omboni S, Parati G, Castiglioni P. Estimation of blood pressure variability from 24-hour ambulatory finger blood pressure. Hypertension 1998; 32: 52-58.

23 Schutte AE, Huisman HW, van Rooyen JM, Malan NT, Schutte R. Validation of the Finometer device for measurement of blood pressure in black women. J Hum Hypertens 2004; 18: 79-84.

24 Guelen I. Finometer, finger pressure measurements with the possibility to reconstruct brachial pressure. Blood Press Monit 2003; 8: 27-30.

25 Van Gestel AJ, Kohler M, Steier J, Teschler S, Russi EW, Teschler H. The effects of controlled breathing during pulmonary rehabilitation in patients with COPD. Respiration 2012; 83: 115-124.

26 Akselrod S, Gordon D, Madwed JB, Snidman NC, Shannon DC, Cohen RJ. Hemodynamic regulation: investigation by spectral analysis. Am J Physiol Heart Circ Physiol 1985; 249: 867-875.

27 Claude J. The enigma of Mayer waves: facts and models. Cardiovasc Res 2006; 70 : 12-21.

28 Chobanian AV, Bakris GL, Black HR, Cushman WC, Green LA, Izzo JL, Jones DW, Materson BJ, Oparil S, Wright JT, Roccella EJ. The Seventh Report of the Joint National Committee on Prevention, Detection, Evaluation, and Treatment of High Blood Pressure: the JNC 7 report. JAMA 2003; 289: 2560-2572.

29 British Cardiac Society, British Hyperlipidaemia Association, British Hypertension Society, British Diabetic Association. Joint British recommendations on prevention of coronary heart disease in clinical practice: summary. BMJ 2000; 320: 705-708.

30 Genuth S, Alberti KG, Bennett P, Buse J, Defronzo R, Kahn R, Kitzmiller J, Knowler WC, Lebovitz H, Lernmark A, Nathan D, Palmer J, Rizza R, Saudek C, Shaw J, Steffes M, Stern M, Tuomilehto J, Zimmet P. Follow-up report on the diagnosis of diabetes mellitus. Diabetes Care 2003; 26: 3160-3167.

31 Clarenbach CF, Stoewhas AC, van Gestel AJR, Latshang TD, Lo Cascio CM, Bloch KE, Kohler M. Comparison of photoplethysmographic and arterial tonometry-derived indices of arterial stiffness. Hypertens Res 2012; 35: 228-233.

32 Chobanian AV, Bakris GL, Black HR, Cushman WC, Green LA, Izzo JL Jr, Jones DW, Materson BJ, Oparil S, Wright JT Jr, Roccella EJ. Seventh Report of the Joint National Committee on Prevention, Detection, Evaluation, and Treatment of High Blood Pressure. Hypertension 2003; 42: 1206-1252.

33 Hansen TW, Thijs L, Li Y, Boggia J, Kikuya M, Björklund-Bodegard K, Richart T, Ohkubo T, Jeppesen J, Torp-Pedersen C, Dolan E, Kuznetsova T, Stolarz-Skrzypek K, Tikhonoff V, Malyutina S, Casiglia E, Nikitin Y, Lind L, Sandoya E, Kawecka-Jaszcz K, Imai Y, Wang J, Ibsen H, O'Brien E, Staessen JA. Prognostic value of reading-to-reading blood pressure variability over $24 \mathrm{~h}$ in 8938 subjects from 11 populations. Hypertension 2010; 55: 1049-1057. 
34 Kikuya M, Hozawa A, Ohokubo T, Tsuji I, Michimata M, Matsubara M, Ota M, Nagai K, Araki T, Satoh H, Ito S, Hisamichi S, Imai Y. Prognostic significance of blood pressure and heart rate variabilities: the Ohasama Study. Hypertension 2000; 38: 23-29.

35 Mancia G, Bombelli M, Facchetti R, Madotto F, Corrao G, Trevano FQ, Grassi G, Sega R. Long-term prognostic value of blood pressure variability in the general population: results of the Pressioni Arteriose Monitorate e Loro Associazioni Study. Hypertension 2007; 49: 1265-1270.

36 Pringle E, Phillips C, Thijs L, Davidson C, Staessen JA, De Leeuw PW, Jaaskivi M, Nachev C, Parati G, O'Brien ET, Tuomilehto J, Webster J, Bulpitt CJ, Fagard RHSystEur investigators. Systolic blood pressure variability as a risk factor for stroke and cardiovascular mortality in the elderly hypertensive population. J Hypertens 2003; 21 2251-2257.

37 Sander D, Kukla C, Klingelhofer J, Winbeck K, Conrad B. Relationship between circadian blood pressure patterns and progression of early carotid atherosclerosis: a 3-year follow-up study. Circulation 2000; 102: 1536-1541.

38 Mancia G, Parati G, Castiglioni P, Tordi R, Tortorici E, Glavina F, Di Rienzo M. Daily life blood pressure changes are steeper in hypertensive than in normotensive subjects. Hypertension 2003; 42: 277-282.

39 Zakopoulos NA, Tsivgoulis G, Barlas G, Papamichael C, Spengos K Manios E, Ikonomidis I, Kotsis V, Spiliopoulou I, Vemmos K, Mavrikakis M, Moulopoulos SD. Time rate of blood pressure variation is associated with increased common carotid artery intima-media thickness. Hypertension 2005; 45 505-512.
40 Manios E, Stamatelopoulos K, Tsivgoulis G, Barlas G, Koroboki E, Tsagalis G, Michas F, Vemmos K, Zakopoulos $N$. Time rate of blood pressure variation: a new factor associated with coronary atherosclerosis. J Hypertens 2011; 29: 1109-1114.

41 Rocco MB, Barry J, Campbell S, Nabel E, Cook EF, Goldman L, Selwyn AP. Circadian variation of transient myocardial ischemia in patients with coronary artery disease. Circulation 1987; 75: 395-400.

42 Mancia G, Frattola A, Parati G, Santucciu C, Ulian L. Blood pressure variability and organ damage. J Cardiovasc Pharmacol 1994; 24 (Suppl. A): 6-11.

43 White WW. Blood pressure monitoring in cardiovascular medicine and therapeutics. Clin Hypertens Vasc Dis 2007; ii: 253-291.

44 Stamatelopoulos KS, Manios E, Barlas G, Koroboki E, Zacharoulis A, Tsivgoulis G, Kollias G, Kyrkou K, Tsigas N, Papamichael CM, Zakopoulos NA. Time rate of blood pressure variation is superior to central hemodynamics as an associate of carotid intima-media thickness. J Hypertension 2010; 28: 51-58.

45 Sorond FA, Khavari R, Serrador JM, Lipsitz LA. Regional cerebral autoregulation during orthostatic stress: age-related differences. J Gerontol A Biol Sci Med Sci 2005; 60 1484-1487.

46 Rothwell PM. Does blood pressure variability modulate cardiovascular risk? Curr Hypertens Rep 2011; 13: 177-186.

47 Lammie GA, Lindley R, Keir S, Wiggam MI. Stress-related primary intracerebral hemorrhage: autopsy clues to underlying mechanism. Stroke 2000; 31: 1426-1428.

48 Meyer JS, Waltz AG, Gotoh F. Pathogenesis of cerebral vasospasm in hypertensive encephalopathy. I. Effects of acute increases in intraluminal blood pressure on pial blood flow. Neurology 1960; 10: 735-744. 